\title{
The Identity Choice of Working Women: Conflict and Integration
}

\section{Weilu Wu}

Nanjing University of Science and Technology, Nanjin 210094, China;

lulu086@163.com

\begin{abstract}
Nowadays, working women not only undertake the same pressure in the work as men, but also need to play the family roles well after work. How to play various roles well and make balance between work and family is a major issue that contemporary working women have to face. Using the empirical research method, this research carried out in-depth interviews of 29 working women, and analyzed the interview data with qualitative research methods. Through introducing the roles of contemporary working women, analyzing the performance, causes, advantages and disadvantages of the role conflict, the purpose of this study is to make suggestions of how to deal with work-family conflicts and seek work-family balance.
\end{abstract}

Keywords: Working women; Qualitative research; Work-Family conflict; Balance

\section{职业女性的身份选择：冲突与整合}

\author{
吴伟璐 \\ (南京理工大学 经济管理学院、企业管理系，南京市 中国)
}

摘要: 目前, 职场女性除了要在工作中承担与男性同等的压力外, 回家后还要扮演好家庭角色。如何扮演好各种角色, 在工作一家庭之间取得平衡, 是当代职业女性不得不面临的一个重大课题。本文采用实证性研究方法, 对29名职业女性展开 有针对性的深入访谈, 并通过质性研究方法对访谈资料进行深入分析, 旨在通过介绍当代职业女性的角色, 剖析角色冲突的 表现、原因及其利害, 并在此基础上针对当代职业女性如何处理工作一家庭矛盾, 寻求平衡提出建议。

关键词: 职业女性; 质性研究; 工作-家庭冲突；平衡

\section{引言}

步入 21 世纪, 在现代文明为主导的社会化大转型中, 面临颠覆的不仅是传统行业格局, 社会分工也 被提出了新的要求, 职业女性这一新型的群体便诞生在这种背景下 [1]。她们突破了传统文化中伦理纲常 对女性的束缚, 不再只满足于家庭中的贤妻良母角色, 她们开始走向社会, 寻求个性的解放和自我人生价 值的实现。女性职业化趋势加大, 越来越多的女性参与社会分工劳动。从角色这一层面来看, 职业女性从 原来贤妻良母的家庭妇女转变为职业化后的双重身份：一为家庭主妇，一为社会角色（或者说是因工作而 获得的社会地位)。同时, 拥有社会性工作的女性, 因经济地位提高、角色冲突而带来的诸多问题, 正是 本文探讨和分析的所在。

\section{1 理论回顾}

\section{1 中国女性的社会身份}

现代女性身份扮演主要有三种模式:（1）传统的女性身份和社会塑形：做一个贤妻良母;（2）新型的 女性身份：和男子一样在那些受人尊敬的领域中功成名就; (3) 双重身份扮演: 承担家庭和事业的双重责 任 [2]。中国女性的社会身份主要包括家庭身份、职业身份及其他身份。

中国传统文化决定了在家庭中女性的从属地位，女性在家庭中所扮演的身份以 “贤妻良母”为典范， 
其身份包括女儿、母亲、姐妹、妻子、儿媳、妯娌等。女性在家庭中竭尽所能肩负着几乎所有的家务, 以 自身努力维系家庭的运转, 在面对家庭与自身利益产生冲突时, 大部分女性会选择牺牲自己而满足家庭的 需求。随着世界范围内女权运动的兴起, 我国女性的地位也日益被重视, 上述现象有所变化, 但女性对家 庭的无私奉献与对家务的无尽责任, 在当今社会中仍是十分普遍的存在。

职业身份是指人们从事相对稳定的、有收入的、专门类别的社会劳动身份 [3]。女性在当今社会的现 代化建设中发挥了不可磨灭的作用, 她们的智慧与创新, 她们在工作中所展现出来的能力并不输男性, 新 的价值观和价值取向使得职业女性有了自主意识，在工作中展现了自己的需求与价值。

除家庭身份与职业身份以外，职业女性还担当着其他身份。如朋友、闺蜜、顾客、乘客、观众等等。 这些纷繁复杂的身份同样需要女性付出时间精力, 但相比于职业身份来说, 它们对职业女性身份冲突的产 生影响并不大。

\section{2 职业女性的身份冲突}

职业女性兼具家庭身份和职业身份，由于社会对不同身份的社会期望和规范不同 [4]，带来了职业女 性特有的身份冲突。

\section{2 .1 身份冲突的具体表现}

职业女性在选择不同身份时, 在时间、空间、身心、能力和行为等方面的不同步、不协调主要表现为 以下几个方面:

(1) 进行职业选择时的身份冲突。大部分女性在职业选择时, 往往把家庭身份作为自己的重要身份。 未婚女性在择业时会考虑职业是否有利于未来的择偶与家庭发展, 已婚职业女性在考虑工作是否理想时, 往往会把对孩子、家庭照料的便利程度、离家的远近等等作为选择的标准 [5]。

（2）身份期望和评价标准带给职业女性的困惑。由于不同角色的角色规范不同，女性多面对的角色期 望也不尽相同。家庭要求女性温存、体贴、贤惠、勤劳、将家务料理得井井有条, 社会又要求女性独立、 勇敢, 将工作干得生机勃勃。不同于男性工作者, 职业女性的成功要受到双重标准的检验 [6], 事业和家 庭的共同成功才会被社会定义为成功。

（3）传统性别身份观念与现代身份意识的冲突。对于当代职业女性来说，一方面她们拥有自主意识， 要实现抱负与价值, 一方面她们依然走不出传统观念的羁绊。职业女性兼有家庭身份与职业身份, 使她们 既要面对新的现实又要背负旧的传统 [7]。

（4）时间与精力的冲突。职业女性对缺乏时间和精力的体会比常人更深一层。通常情况下，职业女性 既想事业成功又想家庭幸福, 她们对自身多重角色的期望值很高, 但在实际生活中做到兼顾事业与家庭两 者并不容易。

（5）职业发展中的矛盾冲突。虽然现今社会，各行各业都出现了表现优异的职业女性，但是我们仍能 感受到女性在择业和晋升过程中受到的歧视 [8]。职业女性不得不比男同事工作更加努力, 表现更加优异。

\section{2 .2 身份冲突的形成原因}

职业女性身份冲突形成的原因主要有以下几方面:

（1）传统观念与现代职业观念的碰撞。一方面, 传统的性别身份观念仍占据重要的位置, 人们把主持 家务、照顾家人作为女性 “义不容辞” 的责任; 另一方面, 随着社会的发展, 竞争日益激烈, 现代的职业 观念鼓励更多的女性拥有自己的职业天地, 实现自身的价值 [9]。 
（2）社会人口和家庭结构变化。随着社会转型和经济接轨，中国人口和家庭结构发生了明显的变化, 人口老龄化、城乡流动等给女性造成了更多的家庭任务，职业女性工作家庭之间的冲突日益明显。

（3）时间和精力的宽乏。一个人的时间、精力是有限的，已婚职业妇女，要当好 “贤妻良母” 已非易 事。若要再做好本职工作, 乃至于有所成就, 更是难上加难。

（4）缺乏有力的家庭支持系统。受传统观念的影响，许多男性一方面希望女性分担经济压力，一方面 又将家务全部推给妻子。职业女性缺乏家庭支持, 面对内外压力, 疲于应付。

（5）现代女性对自我的要求和价值追求。现代女性既想通过自己的辛苦劳作，把家庭建成一个温馨的 爱巢, 又想通过参加社会劳动, 获得精神满足与自我价值的实现, 导致她们精疲力尽。

\section{2 .3 身份冲突的利与害}

职业女性的身份冲突是把双刃剑, 有利有害。

身份冲突问题若不加以解决, 必然产生危害: 有损于女性的身心健康发展; 引起家庭矛盾的产生; 不 利于女性的个人发展; 不利于女性幸福感的提升等, 影响女性的生活质量。

然而职业女性的家庭、工作身份冲突也有着其积极的一面。这种矛盾的出现呼唤社会创造更好的物质 和精神条件帮助女性解决这一矛盾, 使女性获得进一步的发展 [10], 与此同时, 社会也将向前迈出一步, 更加完善。

\section{2 研究方法}

本文采用深入访谈形式的质性研究方法。所谓质性研究, 是以研究者本人作为研究工具, 在自然情境 下, 采用多种资料收集方法, 对研究现象进行深入的整体性探究, 从原始资料中形成结论和理论, 通过与 研究对象互动, 对其行为和意义建构获得解释性的理解的一种活动 [11]。针对研究问题, 本文将访谈对象 定位为 1980-1995 年出生的职业女性, 其中以已经结婚生子, 建立自己家庭的职业女性为主。最终, 对 29 名女性进行了深入访谈, 了解工作在职业女性心中的意义、职业女性对工作和家庭的关系的看法、以及其 面对工作家庭身份冲突时的身份选择和整合。

\section{3 研究发现}

\section{1 中国女性的社会身份}

在被访的 29 名女性中，存在 7 种身份，按照提及率分别是妻子 $65.5 \%$ 、母亲 $55.2 \%$ 、员工 $51.7 \%$ 、新 时代女性 $48.3 \%$ 、专业身份 $31.0 \%$ 、女儿 $24.1 \%$ 、创业者 $10.3 \%$, 其中, 属于家庭身份的有妻子、母亲、女 儿，属于职业身份的有员工、创业者，属于其他身份的有新时代身份、专业身份。

通过对被访者的访谈内容进行整理, 我们发现, 对于女性而言, 工作除了有取得一定经济收入这一基 本的意义之外, 还有证明女性自己的能力和独立性的意义。参与工作一方面能够让女性实现自己的自我价 值和人生价值, 在自我提升中获得成就和快乐, 另一方面, 女性也希望通过参与工作能够接触社会, 跟社 会融洽, 并且充实自己，让自己在家庭之外还拥有自己的空间。

\section{2 职业女性的身份冲突}

82. $8 \%$ 的被访者都表示其身份之间有冲突，提及的身份冲突主要包括：妻子身份与职业身份的冲突、 母亲身份与职业身份的冲突、女儿身份与职业身份的冲突。表现为：家庭生活琐事无法及时处理、孩子的 看管和教育难以安排、家人对其工作时间、地点等有意见。原因主要有: 工作时间不够灵活、请假难、出 
差和加班较多等等。造成的后果是家人对其存在不满, 没有足够的时间参与孩子的教育和成长、家庭生活 不和谐。

\section{3 职业女性的身份整合}

面对上述冲突，在有身份冲突的被访者中，63\%的被访者表示出现冲突时会以家庭为主，仅有 $17 \%$ 的 被访者明确表示会以工作为主, 而 $17 \%$ 的女性会根据事情的重要性, 看具体的冲突对哪方的伤害更大来做 出取舍, 或者折中处理。而在目前尚未面临过身份冲突的被采访者中, 50\%的被访者明确表示, 如果有冲 突会首先考虑家庭, 会为了家庭把工作放开。

谈及身份的选择，在 16 位拥有母亲身份的女性中有 11 位选择了母亲身份，她们认为“孩子是生活的 支柱”、“有了孩子之后, 什么都以孩子为重” “孩子小的时候的生活环境很重要”。

\section{4 讨论与结论}

\section{1 职业女性身份整合的内涵与意义}

职业女性进行身份整合时, 最需要关注的是 “工作一生活平衡” , 即正确认识和看待工作和生活之间的 关系, 调和职业和家庭的矛盾, 缓解由于工作生活关系失衡而造成的压力, 兼顾工作与生活。

做好身份整合是职业女性追求工作-家庭的平衡需要。同时，工作-家庭冲突影响员工绩效，组织要提 高员工工作绩效, 就有必要探求工作-家庭平衡方法, 实施平衡策略。此外, 企业实施工作-家庭平衡策略 可成为组织竞争优势, 吸引高素质女性人才。

\section{2 职业女性进行身份整合的方法}

（1）提倡新型的性别角色观念。传统的性别角色概念是造成职业女性角色冲突的一个重要原因, 只有 改变传统的角色定型教育, 提倡新型的男女平等、协调发展的角色教育观念, 角色教育与女性发展才能形 成良性循环。做到这一点, 需要全社会的共同努力。

（2）形成强大的社会與论，鼓励女性走向社会。当代女性在激烈的社会竞争中往往处于劣势，最容易 成为裁员、下岗的首选对象。社会舆论不能大肆鼓吹女性为家庭牺牲事业的自我牺牲精神, 也不能把减轻 社会就业压力的责任强加在女性身上。应力使女性能够广泛就业、高层次就业, 取得和男性同等的地位与 权利, 得到真正的解放。

（3）社会为职业女性创造宽松环境, 使之施展才华。虽然时代在发展, 但女性还是在就业时面对着 “歧 视” 。社会要为职业女性的双重角色统一创造条件, 首先需要国家通过完善立法, 规范男女公平就业的权 利。其次, 要从法律层面加大保障女性平等就业权的力度, 进一步优化政策环境, 保证女性在职场竞争中 不受性别歧视与限制。三是要健全妇女生育的社会保障和补偿机制, 彻底打消用人单位在对女性录用时存 在的对女性育儿的潜在抵抗心理, 给职业女性以工作安全感。

（4）企业实施职业女性身份整合平衡的策略。企业应当制定有利于员工的家庭友好项目，支持和帮助 员工有效处理家庭问题, 如: 进行休假项目, 适当减轻员工工作负荷; 为女性职员提供儿童看护、老年人 护理等服务, 使员工安心工作; 引入员工援助计划, 通过专业人员对女性员工及其家属提供心理咨询、治 疗服务等解决家庭、法律、医疗和经济问题等。

（5）缓解职业女性的角色冲突的一个根本方法就是家务劳动社会化、家庭设施现代化。家人之间应进 行适当的劳动分工, 利用现代化的家庭设施如洗衣机、微波炉、吸尘器等, 分担女性的家务劳作, 并将抚 
养、赡养功能逐渐向社会转移, 如幼儿进托儿所, 老人进敬老院等 [12]。通过将传统的家庭服务功能让位 于社会，让正规化的社区家政服务走进家庭，减轻职业女性的家务劳动负担。

（6）提高职业女性素质，提高女性扮演双重角色的信心。知识经济时代的到来要求新时期的职业女性 的教育水平要得到更高层次的提高 [13], 职业女性应通过各种途径提高自己的文化素质, 锻炼自己扮演各 种角色所需的技能, 在有目的的学习下和对自身职业道德和家庭美德的教育下, 增强扮演双重角色的信心, 从容面对。

（7）面对角色冲突产生的矛盾与压力，需要职业女性自身学会自我解压、自我调试。职业女性应在面 对难以调和的冲突时确立角色代偿意识, 适时调整期望值, 量力而行, 通过确定可实现的目标, 合理安排 工作，减少角色冲突带来的危害。

\section{5 结语}

莎土比亚在《皆大欢喜》中这样写到：“全世界是一个大舞台，所有的男男女女不过是一些演员；你 们都有下场的时候，也有上场的时候，一个人扮演着好几个角色。”

当代的职业女性在扮演着家庭和工作角色同时，面临着角色冲突所带来的前所未有的挑战。通过对角 色冲突进行分析，我们探究总结出促进工作一家庭平衡的各项办法，通过社会、企业和职业女性自身的共 同努力，多管齐下，寻求平衡。

其实, 对于职业女性来说, 家庭与事业并不是二选一的选择题, 而是可以协调共存的, 一个独立坚强、 成熟聪慧的职业女性能够使家庭角色与职业角色协调统一, 在角色冲突中找到平衡, 更加丰富、全面地发 展。

\section{参考文献:}

[1] Sacharin V, Lee F, Gonzalez R. Identities in harmony: gender-work identity integration moderates frame switching in cognitive processing. [J]. Psychology of Women Quarterly, 2009, 33(3):275-284.

[2] Kreiner G E, Sheep M L. Where is the 'me' among the 'we'? Identity work and the search for optimal balance[J]. Academy of Management Journal, 2006,49(5):1031-1057.

[3] Leonardelli G J. Perceiving expatriate coworkers as foreigners encourages aid: social categorization and procedural justice together improve intergroup cooperation and dual identity. [J]. Psychological Science, 2011, 22(1):110.

[4] Lee N, Zvonkovic A M, Crawford D W. The Impact of Work-Family Conflict and Facilitation on Women's Perceptions of Role Balance [J]. Journal of Family Issues, 2014, 35(9):1252-1274.

[5] Laurijssen I, Glorieux I. Career Trajectories for Women After Childbirth: Job Quality and Work-Family Balance[J]. European Sociological Review, 2013, 29(3):426-436.

[6] Leineweber C, Baltzer M, Magnusson Hanson L L, et al. Work-family conflict and health in Swedish working women and men: a 2-year prospective analysis [J]. European Journal of Public Health, 2013, 23(4):710-716.

[7] 金窗爱, 金喜在. 女性职业发展的阻碍因素分析及对策建议[J]. 甘肃社会科学, 2012(1):161-164.

[8] 武晓伟. “双面胶”与“双面焦”— 80 后女性家庭与职业选择困境的研究[J]. 中国青年研究, 2016(11):81-86.

[9] 于维娜, 樊䎣, 张克勤. 职业女性工作一家庭促进和积极行为:心理资本与人一工作匹配的作用 [J]. 经济管理, 2015(3):89-97.

[10] 邓子鹃，林仲华. 国内职业女性工作一家庭冲突研究述评[J]. 妇女研究论从, 2012(2):103-108.

[11] Malterud K. Qualitative research: standards, challenges, and guidelines. [J]. The lancet, 2001,358(9280):483-488.

[12] 杨菊华. 健全托幼服务 推动女性工作与家庭平衡[J]. 妇女研究论从, 2016(2):11-14.

[13] Kibelloh M, Bao Y. Can Online MBA Programmes Allow Professional Working Mothers to Balance Work, Family, and Career Progression? A Case Study in China [J]. The Asia-Pacific Education Researcher, 2014, 23(2):249-259. 


\section{References}

[1] Sacharin V, Lee F, Gonzalez R. Identities in harmony: gender-work identity integration moderates frame switching in cognitive processing. [J]. Psychology of Women Quarterly, 2009, 33(3):275-284.

[2] Kreiner G E, Sheep M L. Where is the 'me' among the 'we'? Identity work and the search for optimal balance [J]. Academy of Management Journal, 2006, 49(5):1031-1057.

[3] Leonardelli G J. Perceiving expatriate coworkers as foreigners encourage aid: social categorization and procedural justice together improve intergroup cooperation and dual identity. [J]. Psychological Science, 2011, 22(1):110.

[4] Lee N, Zvonkovic A M, Crawford D W. The Impact of Work-Family Conflict and Facilitation on Women's Perceptions of Role Balance [J]. Journal of Family Issues, 2014, 35(9):1252-1274.

[5] Laurijssen I, Glorieux I. Career Trajectories for Women after Childbirth: Job Quality and Work-Family Balance [J]. European Sociological Review, 2013, 29(3):426-436.

[6] Leineweber C, Baltzer M, Magnusson Hanson L L, et al. Work-family conflict and health in Swedish working women and men: a 2-year prospective analysis [J]. European Journal of Public Health, 2013, 23(4):710-716.

[7] Chuangai Jin, Xizai Jin. Women's career development obstacles analysis and countermeasures and Suggestions [J]. Gansu social science, 2012(1):161-164.

[8] Xiaowei Wu. "shuang mian jiao"and "shuang mian jiao"—— the generation after 80 s women's family and career choice dilemma [J]. The China youth research, 2016(11):81-86.

[9] Weina Yu, Yun Fan, Keqin Fang. Work-Family Promotion and Positive Behavior of Professional Women: The Role of Psychological Capital and Person-Job Matching [J]. Economic Management Journal, 2015(3):89-97.

[10] Zijuan Deng, Zhonghua Lin. A Review of Chinese Studies on Female Employee's Work-Family Conflict [J]. Collection of Women's Studies, 2012(2):103-108.

[11] Malterud K. Qualitative research: standards, challenges, and guidelines [J]. The lancet, 2001, 358(9280):483-488.

[12] Juhua Yang. Perfecting the Child-care Service to Promote the Work - Family Balance [J]. Collection of Women's Studies, 2016(2):11-14.

[13] Kibelloh M, Bao Y. Can Online MBA Programmes Allow Professional Working Mothers to Balance Work, Family, and Career Progression? A Case Study in China [J]. The Asia-Pacific Education Researcher, 2014, 23(2):249-259. 